\title{
Jamming Proteins with Slipknots and Their Free Energy Landscape
}

\author{
Joanna I. Sułkowska, ${ }^{1}$ Piotr Sułkowski, ${ }^{2,3,4}$ and José N. Onuchic ${ }^{1}$ \\ ${ }^{1}$ Center for Theoretical Biological Physics, University of California San Diego, Gilman Drive 9500, La Jolla, California 92037, USA \\ ${ }^{2}$ Physikalisches Institute and Bethe Center for Theoretical Physics, Universität Bonn, Nussallee 12, 53115 Bonn, Germany \\ ${ }^{3}$ California Institute of Technology, Pasadena, California 92215, USA \\ ${ }^{4}$ Institute for Nuclear Studies, Hoża 69, 00-681 Warsaw, Poland
}

(Received 7 July 2009; published 29 December 2009)

\begin{abstract}
Theoretical studies of stretching proteins with slipknots reveal a surprising growth of their unfolding times when the stretching force crosses an intermediate threshold. This behavior arises as a consequence of the existence of alternative unfolding routes that are dominant at different force ranges. The existence of an intermediate, metastable configuration where the slipknot is jammed is responsible for longer unfolding times at higher forces. Simulations are performed with a coarse-grained model with further quantification using a refined description of the geometry of the slipknots. The simulation data are used to determine the free energy landscape of the protein, which supports recent analytical predictions.
\end{abstract}

DOI: 10.1103/PhysRevLett.103.268103

The large increase in determining new protein structures has led to the discovery of several proteins with complicated topology. This new fact has raised the question if their energy landscape and the folding mechanism is similar to typical proteins. One class of such proteins includes knotted proteins which comprise around $1 \%$ of all structures deposited in the PDB database [1-3]. A related class of proteins contains more subtle geometric configurations called slipknots [4,5]. Recent theoretical studies using structure-based models (where native contacts are dominant) suggest that slipknotlike conformations act like intermediates during the folding of knotted proteins [6]. This entire new mechanism is consistent with energy landscape theory (FEL) and the funnel concept $[7,8]$. It was shown that the slipknot formation reduces the topological barrier. Complementing regular folding studies, additional information about the landscape was obtained by mechanical manipulation of the knotted protein with atomic force microscopy [9] both experimentally in [10,11] and theoretically in [12-14]. For example, it has been shown [12] that unfolding proceeds via a series of jumps between various metastable conformations, a mechanism opposite to the smooth unfolding in knotted homopolymers.

Motivated by these early results, we now propose a unified picture for the mechanical unfolding of proteins with slipknots. In this Letter this question is addressed by explaining the role of topological barriers along their mechanical unfolding pathways. Supported by our previous results that knotted proteins can still have a minimally frustrated funnel-like energy landscape, structure-based theoretical coarse-grained models are used [15] to analyze the behavior of a slipknot protein under stretching. Studies are performed for the $\alpha / \beta$ class protein thymidine kinase (PDB code: 1E2I [16]).

Most of our analysis is based on stretching simulations under constant force [17]. The crucial signature for this process is the overall unfolding time from the beginning of
PACS numbers: 87.15.ap, 82.37.Gk, 87.14.E-, 87.15.La

the stretching until the protein fully unfolds. Normally one expects that the transition between the native and the unfolded basins to be limited by overcoming the free energy barrier, which gets effectively reduced upon an application of a stretching force. The rate by which this barrier is reduced depends on the distance between the unfolded basin and the top of the barrier measured along the stretching coordinate $x$. This idea was first developed in the phenomenological model of Bell [18], which states that the unfolding time $\tau$ decreases exponentially with applied stretching force $F$ as $\tau(F)=\tau_{0} e^{-\left(F x / k_{B} T\right)}$. A refined analysis performed in Ref. [19] revealed that this dependence is more complicated but still monotonically decreasing.

The unfolding times for 1E2I measured in our simulations are shown as the solid red curve in Fig. 1. In contrast to the above expectations, increasing the force in the range $3-3.5 \epsilon / \AA$ surprisingly results in a larger stability of the protein. $\epsilon$ is the typical effective energy of tertiary native contacts that is consistent with the value $\epsilon / \AA \simeq 71 \mathrm{pN}$ derived in [15]. A solution for this paradox is accomplished

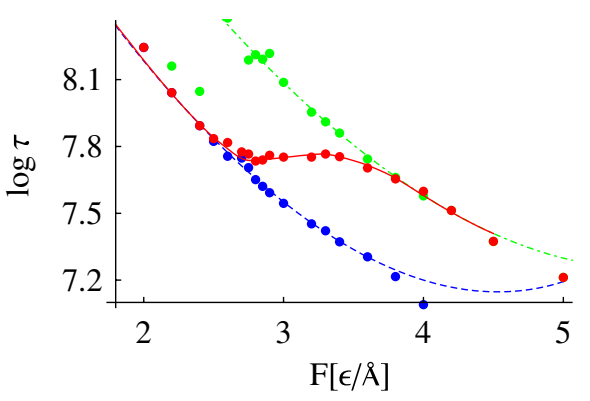

FIG. 1 (color online). Dependence of the unfolding times $\tau$ on the stretching force $F$ for 1E2I (solid line, in red). In this Letter we describe this mechanism as a superposition of two unfolding pathways: I for small forces [dashed (lower) line, in blue], and II for intermidiate and large forces [dash-dotted (upper) line, green]. 
by realizing that unfolding is dominated by two distinct, alternative routes that are dominant at different force regimes. A routing switch occurs when threshold is crossed between weak and intermediate forces. At higher forces, mechanical unfolding is dominated by a route that involves a jammed slipknot. This jamming gives rise to the unexpected dependence of unfolding time on applied force. Characterizing this mechanism is the central goal of this Letter.

To describe the evolution of a slipknot quantitatively requires a refined description. A slipknot is characterized by the three points shown in Fig. 2. The first point $k_{1}$ is determined by eliminating amino acids consecutively from one terminus until the knot configuration is reached (which can be detected, e.g., by applying the KoniarisMuthukumar-Taylor algorithm [20]). The two additional points, $k_{2}$ and $k_{3}$, correspond to the ends of this knot. In the native state the protein 1E2I contains a slipknot with $k_{1}=$ $10, k_{2}=128, k_{3}=298$. These three points divide the slipknot into two loops, which are called the knotting loop and the threaded loop. The former one is the loop of the trefoil knot and the latter one is threaded through the knotting loop. Unfolding of the slipknot upon stretching depends on the relative shrinking velocity of these two loops (see Fig. 3). When the threaded loop shrinks faster than the knotting loop, the slipknot unties. In the opposite case the slipknot gets (temporarily) tightened or jammed, resulting in a metastable state associated with a local minimum in the protein's FEL. Upon further stretching, this configuration eventually also unties. The evolution of both loops of the slipknot is encoded in the time dependence of the points $k_{1}, k_{2}, k_{3}$, see Fig. 3 .

Before discussing the stretching of 1E2I, we explain why a slipknot formed by a uniformly elastic polymer should smoothly unfold under stretching. To simplify the discussion we approximate the threaded and knotting loops by circles of size $R_{t}$ and $R_{k}$. These two loops shrink during stretching and, when the threaded one eventually vanishes, the slipknot gets untied. If both loops have similar sizes, the slipknot is very unstable and unties immediately. When the threaded loop is much larger than the knotting one, $R_{t} \gg R_{k}$, loosening can be explained as follows. The elastic energy associated to local bending is proportional

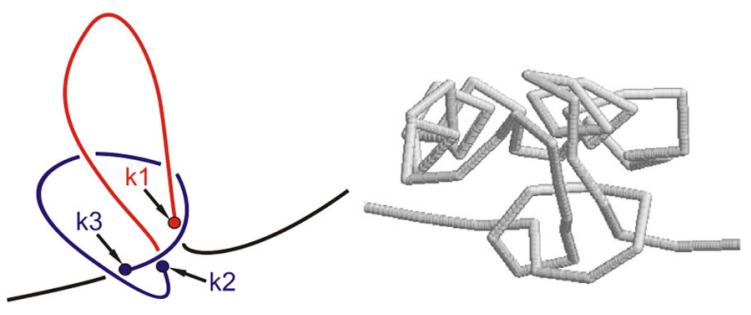

FIG. 2 (color online). A slipknot (left) consists of a threaded loop $\left(k_{1}-k_{2}\right.$, in red or gray) which is partialy threaded through a knotting loop $\left(k_{2}-k_{3}\right.$, in blue or dark gray). An example of a protein configuration with a tightened slipknot is shown in the right panel to the square of the curvature. If the loop is approximated by a circle of radius $R$, then its local curvature is constant and equals $R^{-1}$. The total elastic energy is $\oint d s R^{-2} \sim R^{-1}$ [21]. From the assumption $R_{t} \gg R_{k}$ we conclude that upon stretching it is energetically favorable to decrease $R_{t}$ rather than $R_{k}$. This happens until both radii become equal and then, just as above, the slipknot gets very unstable and loosens. In this discussion we have not yet taken into account that when a slipknot is stretched some parts of a chain slide along each other. This effect could be incorporated by including the friction generated by the sliding [22]. But in the slipknot the sliding region associated with the knotting loop is much longer than the region associated to the threaded loop. Thus this effect results in a faster tightening of the threaded rather than the knotting loop, facilitating even more the loosening of the slipknot.

The above argument should apply to slipknots in biomolecules because they are characterized by a persistence length that in principle is simply related to their elasticity [23]. For DNA this effect is described by wormlike-chain models (WLC) [24] and it has been confirmed experimentally. Although WLC models are too simple to describe the protein general behavior, they are useful in some limited applications. Thus at first sight one might expect that slipknots in proteins should smoothly untie upon stretching. Proteins, however, are much more complicated than DNA or uniformly elastic polymers. The presence of stabilizing native tertiary contacts leads to a jumping charac-

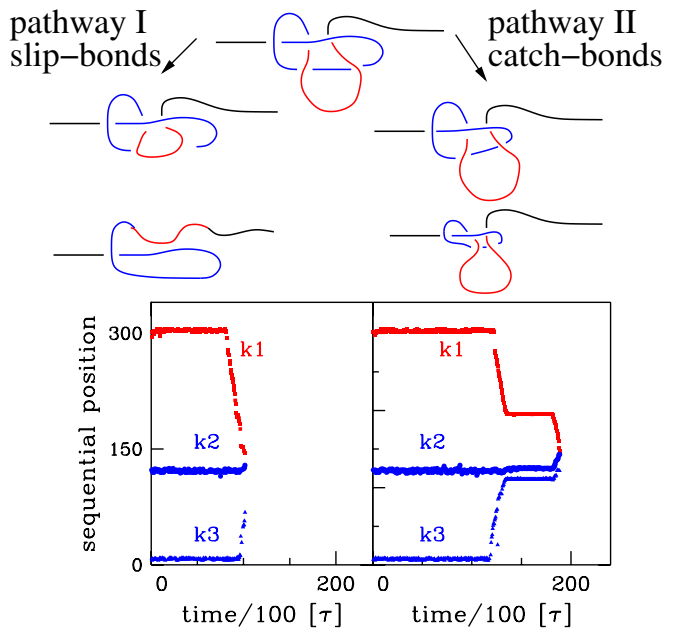

FIG. 3 (color online). The behavior of the slipknot during stretching (top) is determined by the relative behavior of its two loops, encoded in the time dependence of $k_{1}, k_{2}$, and $k_{3}$ (bottom). If the threaded loop shrinks faster than the knotting loop, $k_{1}$ merges with $k_{2}$ (bottom left) and the slipknot loosens (pathway I, top left). If the knotting loop shrinks faster, $k_{2}$ approaches $k_{3}$ (bottom right, $\simeq 14000 \tau$ ) and the slipknot gets temporarily tightened (pathway II, top right). This is a metastable state which can eventually untie upon further stretching, with $k_{1}$ finally merging with $k_{2}$ (bottom right, $\simeq 19000 \tau$ ). Kinetic studies were performed slightly above folding temperature using overdamped Langevin dynamics with typical folding times of $10000 \tau$. 
ter during stretching [12]. In addition their bending energy is not uniform along the chain due to the heterogeneity of the amino-acid sequence. As a consequence it turns out that the intuition obtained through the above analysis of polymers or WLC models is misleading.

Our analysis of the evolution of the endpoints $k_{1}, k_{2}, k_{3}$ (Fig. 3, bottom) reveals that for various stretching forces unfolding proceeds along two distinct pathways (Fig. 3, top). In pathway I the slipknot smoothly unties, which is observed for relatively weak forces. At intermediate forces pathway II starts to dominate and the knotting loop can shrink tightly before the threaded one vanishes. In this regime the protein gets temporarily jammed (Fig. 3, right), leading to much longer unfolding times (catch pathway). The probability of choosing pathway I at different forces is shown in Fig. 4. This pathway competition explains the nontrivial total unfolding time dependence observed in Fig. 1.

The two different pathways I and II arise from completely different unfolding mechanisms. Pathway I starts and continues mostly from the $C$-terminal side, along $16 \alpha$, $15 \beta, 14 \alpha, 13 \beta, 12$ (helices bundle), $11 \alpha$ (here the number denotes a consecutive secondary structure as counted from $N$ terminal, and $\alpha$ or $\beta$ specifies whether this is a helix or a $\beta$ sheet; for more details about the structure of 1E2I see the PDB). This is followed by unfolding of helices $11 \alpha, 10 \alpha$ that allows breaking of the contacts inside the $\beta$ sheet created by the $N$ terminal, with unfolding proceeding also from the $N$ terminal. Pathway II also starts from the $C$ terminal but rapidly (as soon as helix 15 is unfolded) switches to the $N$ terminal. In this case, differently from pathway I, the $\beta$ sheet from the $N$ terminal unfolds even before $13 \beta$. These scenarios indicate that the pathway I should be dominant at weak forces since they are not sufficient to break the $\beta$ sheet during the first steps of unfolding. The jammed pathway is typical only if stretching forces are sufficiently strong for unfolding to proceed from the two terminals of the protein.

A similar phenomenon was firstly proposed in Ref. [25] and referred to as catch bonds. Experimental evidence sug-

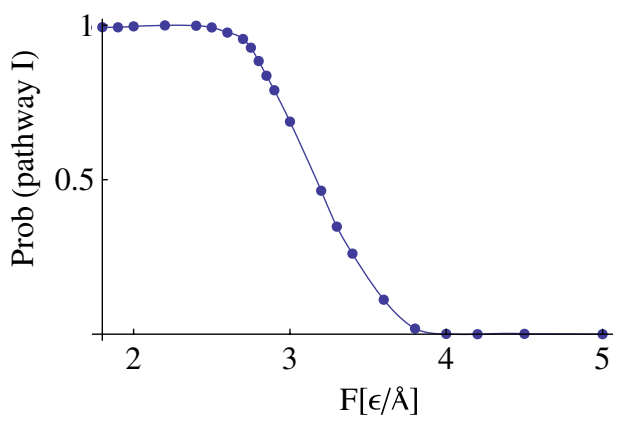

FIG. 4 (color online). Dependence on the applied stretching force of the probability of choosing pathway I rather than II (see Fig. 3). This varying probability leads to the complicated dependence of the total unfolding time on the stretching force observed in Fig. 1. gesting this mechanism was first observed for adhesion complexes [26,27]. Using atomic force microscopy (AFM), at large forces the ligand-receptor pair becomes entangled and therefore expands the unfolding time. A theoretical description of this mechanism was given in Refs. [28-30].

The kinetic data can also be used to determine the associated free energy landscape (FEL) [7]. In an initial simplification we associate the barriers along the stretching coordinate as the kinetic bottlenecks during the mechanical unfolding event. Generalizing Bell's model, a recent description of two-state mechanical unfolding in the presence of a single transition barrier has been developed in [19], with the rate equation

$$
\tau(F)=\tau_{0}\left(1-\frac{\nu F x^{\dagger}}{\Delta G}\right)^{1-1 / \nu} e^{-\left(\Delta G / k_{B} T\right)\left(1-\left(1-\nu F x^{\dagger} / \Delta G\right)^{1 / \nu}\right)}
$$

where $\nu$ encodes the shape of the barrier. Here $x^{\dagger}$ denotes the distance between the barrier and the unfolded basin (in a first approximation it can be regarded as $F$ independent) and lies on the reaction coordinate along the AFM pulling direction. It can be experimentally determined by measuring how the stretching force modulates the unfolding times $\tau$. The height of the barrier is denoted by $\Delta G$. Figure 1 (unfolding times are given by solid red line) shows that this single barrier theory is not sufficient for the full range of forces. As described before, in the higher force regime, additional basins have to be included in the energy landscape. Models with several metastable basins have been called multistate FEL models [31]. Evidence supporting the need of multistates FEL was confirmed by AFM experiments in different systems $[32,33]$.

To construct a multistate FEL that incorporates two unfolding pathways I and II we use a linear combination of Eq. (1)-like expressions with different shapes and barrier heights. Each one of them essentially accounts for the distinct barrier along a relevant unfolding route. Fitting the stretching data to Eq. (1) with a cusplike $\nu=1 / 2$ approximation (another possibility $\nu=2 / 3$ for the cubic potential in general leads to similar results [19]) determines accurately the location and the height of the potential barriers. Pathway II involves two barriers: first until the moment of creation of the intermediate which is followed the untieing event. They are characterized by $\left(x_{1}, \Delta G_{1}\right)$ and $\left(x_{2}, \Delta G_{2}\right)$ arising, respectively, from the lower and upper fits in Fig. 5 (left). The superposition of these two fits gives the overall mean unfolding time for pathway II [dotdashed (upper) green curve in Fig. 1]. For the ordinary slipknot unfolding (pathway I), the results $x_{I}$ and $G_{I}$ arise from the dashed blue curve in Fig. 1. This analysis leads to the results

$$
\begin{array}{lll}
x_{1}=2.3 \frac{k_{B} T \AA}{\epsilon}, & x_{2}=0.7 \frac{k_{B} T \AA}{\epsilon}, & x_{I}=1.4 \frac{k_{B} T \AA}{\epsilon}, \\
\Delta G_{1}=8.0 k_{B} T, & \Delta G_{2}=4.2 k_{B} T, & \Delta G_{I}=4.7 k_{B} T .
\end{array}
$$

We conclude that the free energy landscape consists of two 


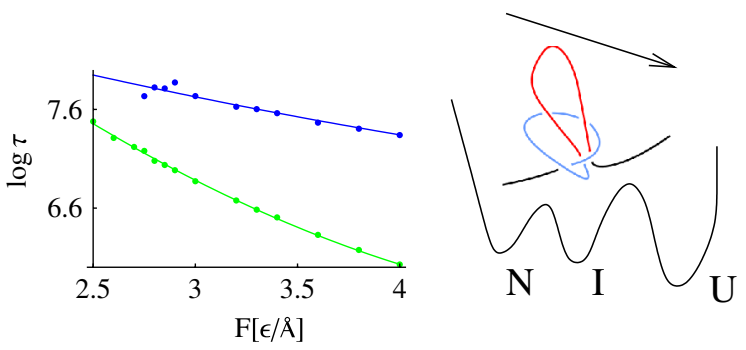

FIG. 5 (color online). Pathway II with two barriers. Left: dependence of the unfolding time on the applied force with the data and the fit to the formula (1) for the first maximum (lower, in green) and for the second maximum (upper, in blue). Right: schematic free energy landscape for this pathway, with jammed slipknot in a minimum between two barriers.

"valleys." The force-dependent probability of choosing one of the valleys during stretching depends on the details of the protein structure. It is determined from our simulations as shown in Fig. 4. Using these probability values and the parameters above for $x$ and $\Delta G$, we can accurately represent the simulation data using a linear combination of equations of the form (1). This agreement supports our analytical analysis and generalizes Eq. (1) for the full of range forces. In addition it demonstrates that structurebased models sufficiently capture the major geometrical properties of a slipknotted protein. A schematic representation of the free energy landscape for pathway II is shown in Fig. 5 (right).

Summarizing, we have analyzed the process of tightening of the slipknot in protein 1E2I and determined the corresponding free energy landscape. Its main feature is the presence of a metastable configuration with a tightened slipknot, which is observed for sufficiently large pulling forces. This phenomenon does not exist for uniformly elastic polymers. In this Letter we concentrated on protein 1E2I but similar behavior has also been observed for other proteins with slipknots, e.g., 1P6X. Our results provide testable predictions that can now be verified by AFM stretching experiments.

We thank T. Deguchi, O. Dudko, K. Millett, and E. Rawdon for insightful discussions. The work of J. S. was supported by the Center for Theoretical Biological Physics sponsored by the NSF (Grant No. PHY-0822283) with additional support from NSF-MCB-0543906. P. S. acknowledges the support of the Humboldt Foundation, DOE Grant No. DE-FG03-92ER40701FG-02, MarieCurie IOF Fellowship, and Foundation for Polish Science.

[1] M. L. Mansfield, Nat. Struct. Biol. 1, 213 (1994).

[2] P. Virnau, L. A. Mirny, and M. Kardar, PLoS Comput. Biol. 2, e122 (2006).

[3] D. Bolinger, J. I. Sułkowska, Hsiao-Ping Hsu, L. A. Mirny, M. Kardar, J. N. Onuchic, and P. Virnau, PLoS Comput. Biol. (to be published).
[4] T. O. Yeates, Todd S. Norcross, and N. P. King, Curr. Opin. Chem. Biol. 11, 595 (2007).

[5] W. R. Taylor, Comp. Biol. Chem. 31, 151 (2007).

[6] J.I. Sułkowska, P. Sułkowski, and J. N. Onuchic, Proc. Natl. Acad. Sci. U.S.A. 106, 3119 (2009).

[7] P. E. Leopold, M. Montal, and J. N. Onuchic, Proc. Natl. Acad. Sci. U.S.A. 89, 8721 (1992).

[8] J. N. Onuchic and P. G. Wolynes, Curr. Opin. Struct. Biol. 14, 70 (2004).

[9] M. Rief, M. Gautel, F. Oesterhelt, J. M. Fernandez, and H. E. Gaub, Science 276, 1109 (1997).

[10] M. T. Alam, T. Yamada, U. Carlsson, and A. Ikai, FEBS Lett. 519, 35 (2002).

[11] T. Bornschloegl, D. M. Anstrom, E. Mey, J. Dzubiella, M. T. Rief, and K. Forest, Biophys. J. 96, 1508 (2009).

[12] J. I. Sułkowska, P. Sułkowski, P. Szymczak, and M. Cieplak, Phys. Rev. Lett. 100, 058106 (2008).

[13] J. I. Sułkowska, P. Sułkowski, P. Szymczak, and M. Cieplak, Proc. Natl. Acad. Sci. U.S.A. 105, 19714 (2008).

[14] J. Dzubiella, Biophys. J. 96, 831 (2009).

[15] J. I. Sułkowska and M. Cieplak, J. Phys. Condens. Matter 19, 283201 (2007).

[16] J. Vogt, R. Perozzo, A. Pautsch, A. Prota, P. Schelling, B. Pilger, G. Folkers, L. Scapozza, and G. E. Schulz, Proteins Struct. Funct. Genet. 41, 545 (2000).

[17] N. D. Socci, J. N. Onuchic, and P. G. Wolynes, Proc. Natl. Acad. Sci. U.S.A. 96, 2031 (1999).

[18] G. I. Bell, Science 200, 618 (1978).

[19] O. K. Dudko, G. Hummer, and A. Szabo, Phys. Rev. Lett. 96, 108101 (2006).

[20] K. Koniaris and M. Muthukumar, Phys. Rev. Lett. 66, 2211 (1991).

[21] L.D. Landau and E. M. Lifshitz, Theory of Elasticiy (Pergamon Press, Oxford, 1986), 3rd ed.

[22] B. Audoly, N. Clauvelin, and S. Neukirch, Phys. Rev. Lett. 99, 164301 (2007); R. Gallotti and O. Pierre-Louis, Phys. Rev. E 75, 031801 (2007).

[23] C. Bustamante, J. F. Marko, E. D. Siggia, and S. Smith, Science 265, 1599 (1994).

[24] C. Bouchiat, M. D. Wang, J.-F. Allemand, T. Strick, S. M. Blockand, and V. Croquette, Biophys. J. 76, 409 (1999).

[25] M. Dembo, D. C. Torney, K. Saxman, and D. Hammer, Proc. R. Soc. B 234, 55 (1988).

[26] B. T. Marshall, M. Long, J.W. Piper, T. Yago, R. P. McEver, and Ch. Zhu, Nature (London) 423, 190 (2003).

[27] W. Thomas, M. Forero, O. Yakovenko, L. Nilsson, P. Vicini, E. Sokurenko, and V. Vogel, Biophys. J. 90, 753 (2006).

[28] D. Bartolo, I. Derenyi, and A. Ajdari, Phys. Rev. E 65, 051910 (2002).

[29] E. Evans, A. Leung, V. Heinrich, and Ch. Zhu, Proc. Natl. Acad. Sci. U.S.A. 101, 11281 (2004).

[30] V. Barsegov and D. Thirumalai, Proc. Natl. Acad. Sci. U.S.A. 102, 1835 (2005).

[31] I. Schwaiger, M. Schleicher, A. A. Noegel, and M. Rief, EMBO J. 6, 46 (2005).

[32] P. M. Williams, S. B. Fowler, R. B. Best, J. L. TocaHerrera, K. A. Scott, A. Stward, and J. Clarke, Nature (London) 422, 446 (2003).

[33] C. Cecconi, E. A. Shank, C. Bustamante, and S. Marqusee, Science 309, 2057 (2005). 